\title{
Study of Aneaka and Cloudsim simulators
}

\author{
Nitin S. More ${ }^{\# 1}$, Dr. R. B. Ingle \#2 $^{\# 2}$ \\ \#1 PICT College, Pune \\ \#2 PICT College, Pune
}

\section{ABSTRACT}

Cloud computing use is increasing day by day as many people are using smart phones, gadgets which are highly computing in nature and for operating them there is need of virtual machines. Cloud computing gaining huge response in market because of daily or we can say per second there is increase in data. For storing and processing them there is best option available i.e. cloud. Virtualization is the basic technique of creating number of options in terms of solutions to the increasing demands from the available limited physical infrastructure. It is the backbone of cloud computing technology. These paper try to deal with studying and evolving various modus operandi, algorithms, and models for proficient green cloud computing by consuming virtualization methods. It mainly involves virtual machines (VMs) consolidation. Power utilization can be reduced by simply deactivating and reactivating physical machines to meet the current workload. By presenting precise study and use of cloud development tools namely Aneaka and Cloudsim.

Thereare many techniquesavailable for implementing cloud scenario, some of them are freely available some open source and hence available free of cost to implement. As implementation of actual cloud required many resources and high band internet, simulation of cloud and its component is the good option of researchers.

Key words:cloud computing, aneaka cloud, cloudsim, cloud simulator, open source, data center, energy efficient, etc.

Corresponding Author:Nitin S. More

\section{INTRODUCTION}

Cloud computing is an advance form of distributed systems, which is evolved in the 90's, but origin was in 1960.The origin of cloud computing comes in the year 1961 by computer scientist John McCarthy he said "If computers of the kind I have advocated become the computers of the future, then computing may someday be organized as a public utility just as the telephone system is a public utility. ... The computer utility could become the basis of a new and important industry." He was the first to think about utility computing and nowadays it is reality, just like any other utility bill every house paying internet bills. Later in 1969, Leonard Kleinrock, a chief scientist of ARPANET(Advanced Research Projects AgencyNetwork) project which is base of world wide web i.e. internet, he said "As of now, computer networks are still in their infancy, but as they grow up and become sophisticated, we will probably see the spread of 'computer utilities'..".

Common man started to utilize computer from mid-1990's through various applications like for office purpose email(Hotmail, Gmail), search engines(Yahoo, Google) for knowledge mining purpose, open publishing platforms (YouTube, Orkut, Facebook) and other various social media (LinkedIn, Twitter). Yet this all applications are consumer centric it gain lots of popularity and form the base of today's cloud computing. 


\section{STUDY OF ANEKA CLOUD}

Aneaka cloud developed by Manjrasoft Pty. Ltd. invented by Dr. RajkumarBuyya was done. Study of Aneka cloud application development platform was carried out along with installation for testing purpose. Aneka is cloud application development platform for study purpose developed by Manjrasoft Pty. Ltd [7] by RajkumarBuyya. It is mostly used for developing testing cloud application and cloud data intensive application. User can built his own cloud application by using it or they can also test, run, modify their already available cloud application code through API's and tools which are available with Aneka.

With Aneka user can build cloud by using his own existing infrastructure, can opt for various deployment options all these facillated by very easy and interactive Aneka cloud Graphical user interface (GUI). AnekaGUI consists of Management Studio, Daemons and Containers.

An Aneka cloud is composed of services which are available on above of all infrastructures available which can consist of local machine or network of machines connected in LAN for creating data center. As illustrated in above figure1 aneka cloud management studio GUI helps you to build cloud this is installed on main machine called as aneka daemon which contains many containers or virtual machines. Aneka cloud administrator equipped with aneka management studio is responsible for managing all cloud infrastructures and cloud as shown in figure 2 aneka cloud management scenario.

Aneka services are achieved by aneka daemons and all this services are hosted by aneka containers, daemon is actually service running in background on strong computer which controlling aneka containers by installing, running, stopping, updating and reconfiguring.

Aneka cloud administrator uses management studio for managing aneka daemons and aneka cloud containers which are hosted by different machines in data center or LAN. Admin install many containers may be on same machine or on sub machines in data center as per the requirement for controlling, configuring various containers.

Every machine in aneka cloud consist of daemons and containers in aneka cloud platform which is managed by master daemon through management studio, this is master daemon responsible for scheduling jobs to worker nodes available in aneka cloud. In aneka cloud each node can run many containers as per the capability. Worker node in aneka cloud which is getting some job from master container may be physical machine or virtual machine. All containers are handled by respective daemon available on that node. The figure 3 given below shows high level view of aneka cloud. Table 1 shows comparison of aneaka programming clouds.

Table1. Different programming modules of Aneaka

\begin{tabular}{|c|c|c|c|c|}
\hline Properties & $\begin{array}{c}\text { Thread } \\
\text { Programming } \\
\text { Model } \\
\end{array}$ & $\begin{array}{c}\text { Task Programming } \\
\text { Model }\end{array}$ & $\begin{array}{c}\text { Map Reduce } \\
\text { Programming } \\
\text { Mode }\end{array}$ & $\begin{array}{c}\text { Parameter } \\
\text { sweeping } \\
\text { Model }\end{array}$ \\
\hline Types & Low level & $\begin{array}{l}\text { Basic general } \\
\text { model }\end{array}$ & High level & $\begin{array}{c}\text { Rapid } \\
\text { prototype }\end{array}$ \\
\hline Operations & $\begin{array}{l}\text { Start, stop state } \\
\text { query and join }\end{array}$ & Submit and forget & $\begin{array}{l}\text { Mao (map::(key1, } \\
\text { value1)= list (key2, } \\
\text { value2) ) } \\
\text { Reduce (reduce: } \\
\text { (key2, list value2)= } \\
\text { list (value3) }\end{array}$ & parallelism \\
\hline
\end{tabular}




\begin{tabular}{|c|c|c|c|c|}
\hline \hline Middleware & No & No & Yes & Yes \\
\hline Usage & $\begin{array}{c}\text { Distributed } \\
\text { network } \\
\text { systems }\end{array}$ & $\begin{array}{c}\text { Legacy } \\
\text { applications }\end{array}$ & $\begin{array}{c}\text { Data mining and } \\
\text { analytics }\end{array}$ & $\begin{array}{c}\text { Legacy } \\
\text { applications }\end{array}$ \\
\hline User domain & Yes & Yes & No & No \\
\hline $\begin{array}{c}\text { Function } \\
\text { based }\end{array}$ & No & No & Yes & Yes \\
\hline Task based & Yes & Yes & No & No \\
\hline Examples & Mandelbrot & $\begin{array}{c}\text { Excel grid, } \\
\text { Convolution }\end{array}$ & Word counter & -- \\
\hline
\end{tabular}

IMPLEMENTAION OF ANEAKA CLOUD

Aneka cloud on one physical machine and with local repository installed and created, two repositories created namely local and Nsm_Repository and two users created aneka figure 1 shows it and anekal with credentials to handle the cloud, screenshots of the same shown in figure 2 and figure 3 respectively. Setup is created on one machine having configuration as Windows XP, 3GB RAM,Intel (R) core 2duo processor with $2.20 \mathrm{GHz}$ Acer aspire 4736 laptop machine.

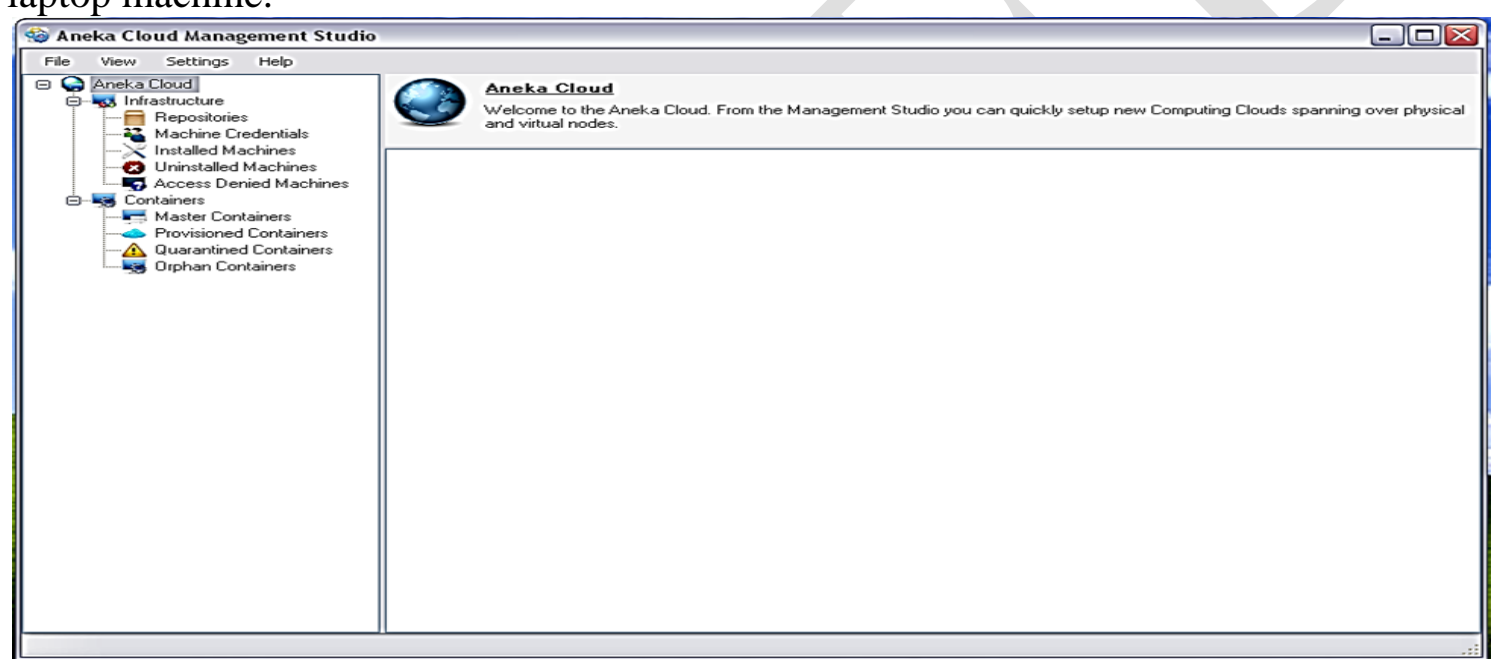

Figure 1. Aneka cloud management studio

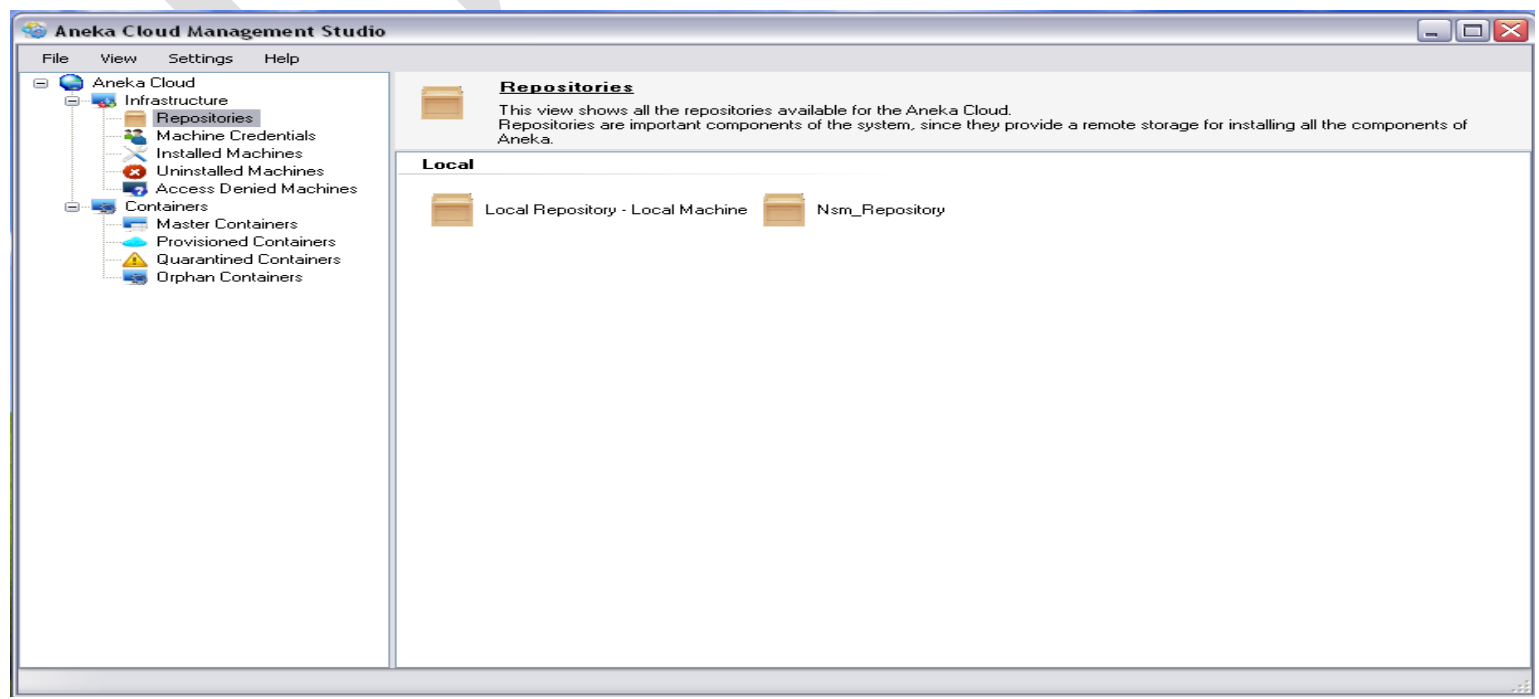

Figure 2 Repositories created in aneka cloud 
Further it required to be tested with relational and non-relational databases for energy optimization. Apart from this one daemon and container created on the same physical machine and aneka cloud test bed created.

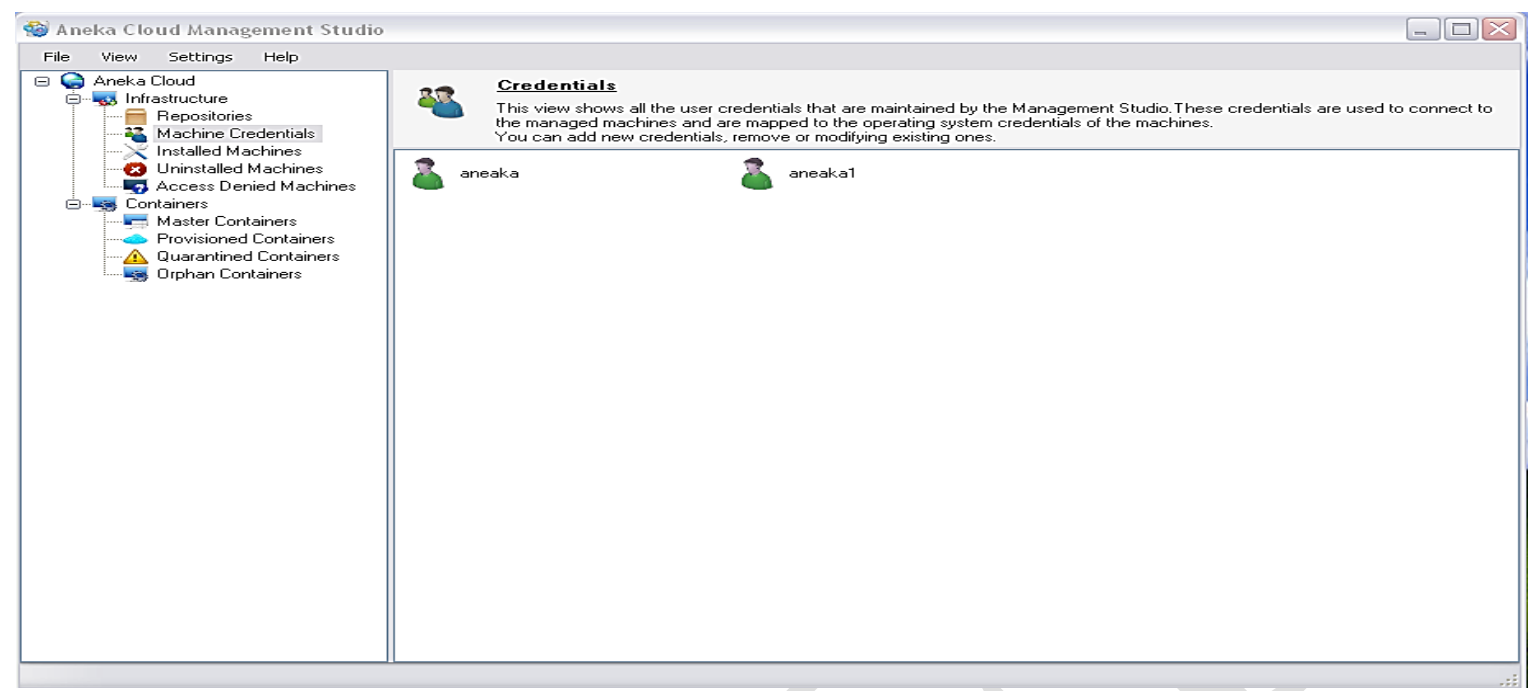

Figure 3 Two users created for aneka cloud

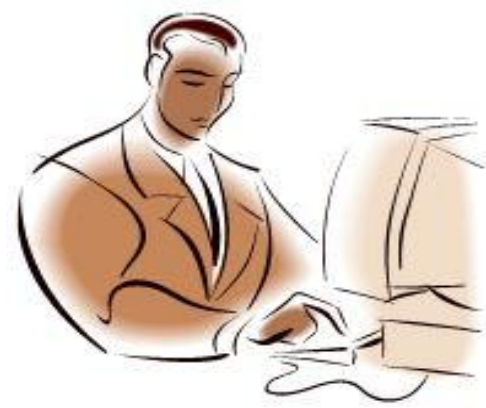

Aneka Cloud Administrator with Aneka management studio
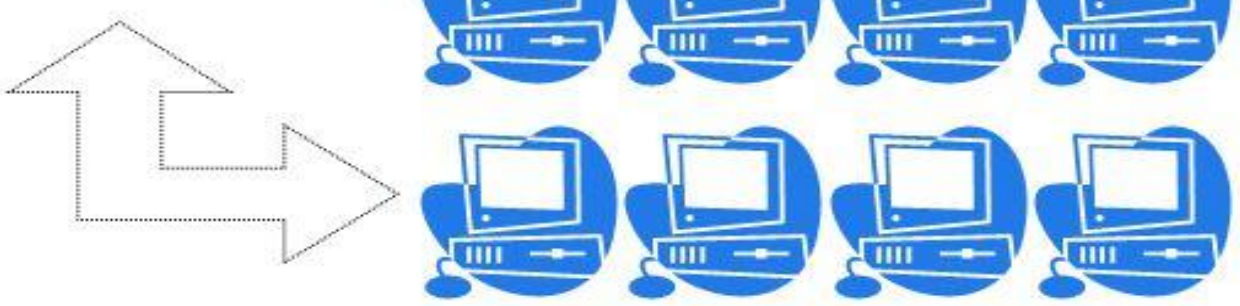

Data Center or LAN hosting

Aneka cloud

Figure4. Aneka cloud management studio architecture

\section{STUDY OF CLOUDSIM}

Study of Aneaka Cloud developed by Manjrasoft Pty. Ltd. invented by Dr. Rajkumar Buyya was done, which was already discussed with previous progress report. Cloudsim 3.0 cloud simulator developed by cloudlabs, Melbourne University, study and implementation of it also carried out for better understanding of implementation of proposed algorithms and test bed scenarios. This progress report discusses the work done with Cloudsim simulator. 
Cloudsim is a simulation toolkit developed by cloudlabs, Melbourne University, Australia under the guidance of Dr. Rajkumar buyya and his team. Cloudsim [1] is developed in Java, it is alike other simulators which are already available like GridSim [9], SimGrid [7], andGangSim but with some other extra features. This all previous simulators are used for grid simulation and cloudsim is developed by extending Gridsim layer. In cloudsim extra features like infrastructure management, virtualization of the resources etc, are considered.

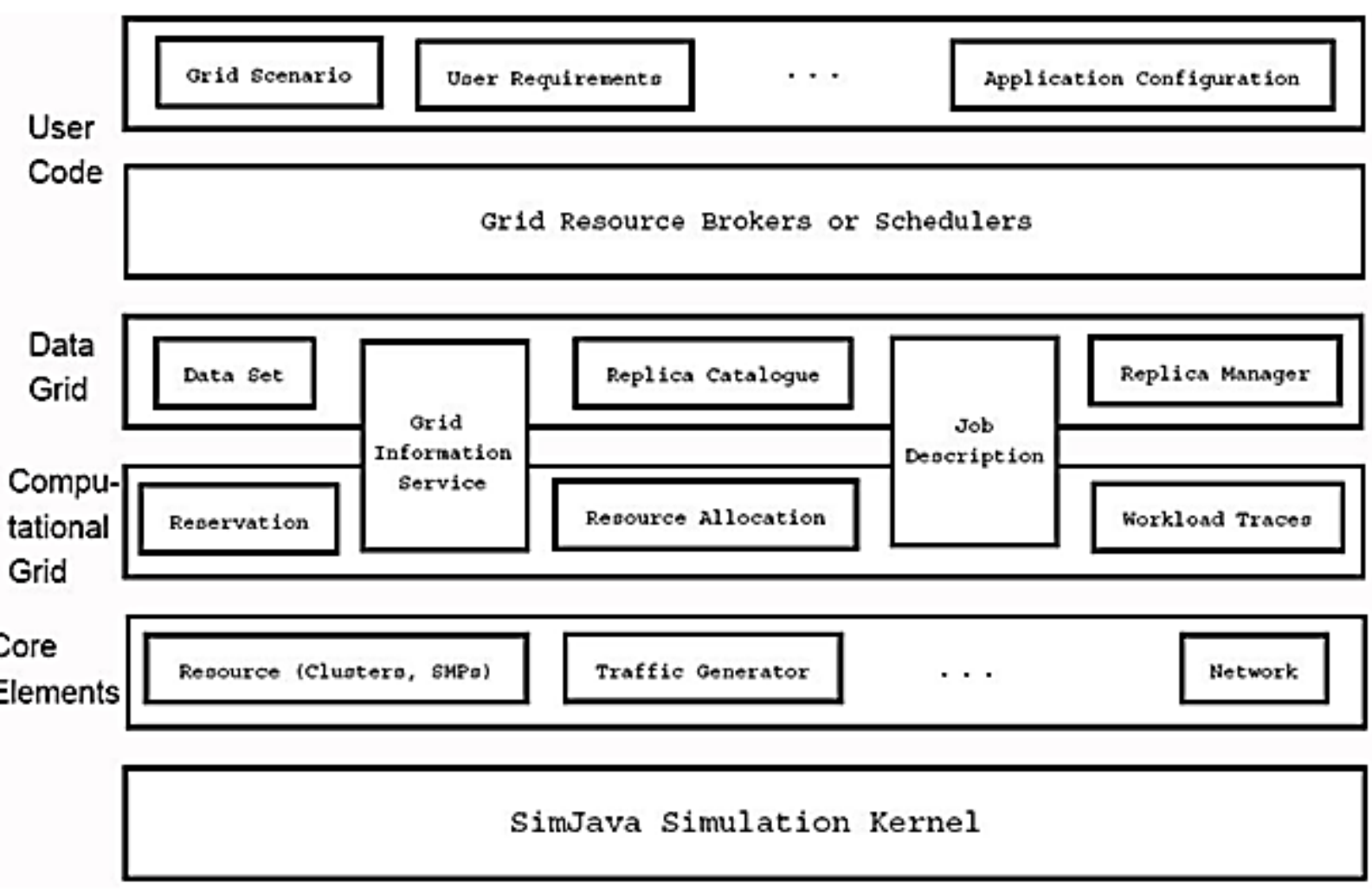

Figure 5 Layered cloudsim architecture [1]

Above figure 5 shows layered cloudsim architecture which is obtained by extending gridsim simulator this helps in testing and building novel algorithms, benchmarking policies for cloud computing paradigm from industrial as well as academia.

\section{MATHEMATICAL MODEL FOR ENERGY CONSUMPTION IN CLOUD}

In previous progress report general mathematical equation proposed for energy consumption during read and write operations. This can be further extended with energy consumption of virtual machine present in cloudas follows,

$$
\begin{gathered}
E_{c}=E_{\text {vmrunning }}+E_{\text {vmmigrating }}+E_{\text {vmidle }} \ldots \ldots \ldots \ldots(I) \\
\text { Where } \mathrm{E}_{\mathrm{vmrunning}}=N_{t} \cdot e_{t}+R_{t} \cdot e_{r t} \ldots \ldots \ldots \ldots(I I) \\
E_{\text {vmmigrating }}=f(\text { nu, pm) } \ldots \ldots \ldots \ldots \ldots \text { (III) } \\
E_{\text {vmidle }}=E_{v m} \text { whent }=0 \ldots \ldots \ldots \ldots \ldots(I V)
\end{gathered}
$$

$E_{c}-$ Energy of cloud, $E_{v m r u n n i n}-$ Energy of virtual machine (vm) when vm is running, $E_{\text {vmmigrating }}$ - Energy of vm while migrating, $E_{\text {vmidle }}-$ Energy of vm when vm is idle in equation I. And further equation II, III and IV elaborates the equation of energy of vm when it is running, migrating and idle respectively. In equation II, III and IV $N_{t}$ - number of task t executing at vm, $e_{t}-$ energy consumed by task t, $R_{t}$-number of resources used during task $\mathrm{t}$, $e_{r t}$-energy consumed by resources during task $\mathrm{t}, n u$ - network utilization, $p m$ - physical 
machine, $E_{v m^{-}}$energy of vm. Equation I says that Energy of cloud is equal to the sum of energy consumed by vm in three states i.e. vm running, vm migrating and when vm is idle. Equation II says energy consumed by vm during running is sum of product of two things, one is the product of number of task and energy required by that task and second is the product of number of resources required for task and energy required by resources like disk, memory, disk, etc. which can be more expressed with different equations. Equation III says energy of vm which is migration is function of energy used by network utilization and physical machine on which vm running. Lastly equation IV stating the energy of vm when task is executing.

\section{EXPERIMENTATION WITH CLOUDSIM}

There are many helpful classes and entities considered in cloudsim for getting the actual cloud computing environment feel. In these regard test bed is created to implement various algorithms and techniques by using clousim simulation toolkit on my own Acer aspire 4736 laptop with 3GB RAM, Intel (R) core 2duo processor with $2.20 \mathrm{GHz}$, Ubuntu 14 operating system, and Eclipse java Integrated development environment.

Various components like cloud broker, Cloud information service (CIS), datacenter for working model of cloudsim. They work in hand to hand for seamless execution of simulation of cloud computing paradigm.

Cloud broker means the actual allocator for resources, this class checks whether requested resources are available in cloud by contacting CIS and datacenter simultaneously. Following are the different experimentation done and executed successfully by going through cloudlabs tutorials and snapshot of the output is provided.

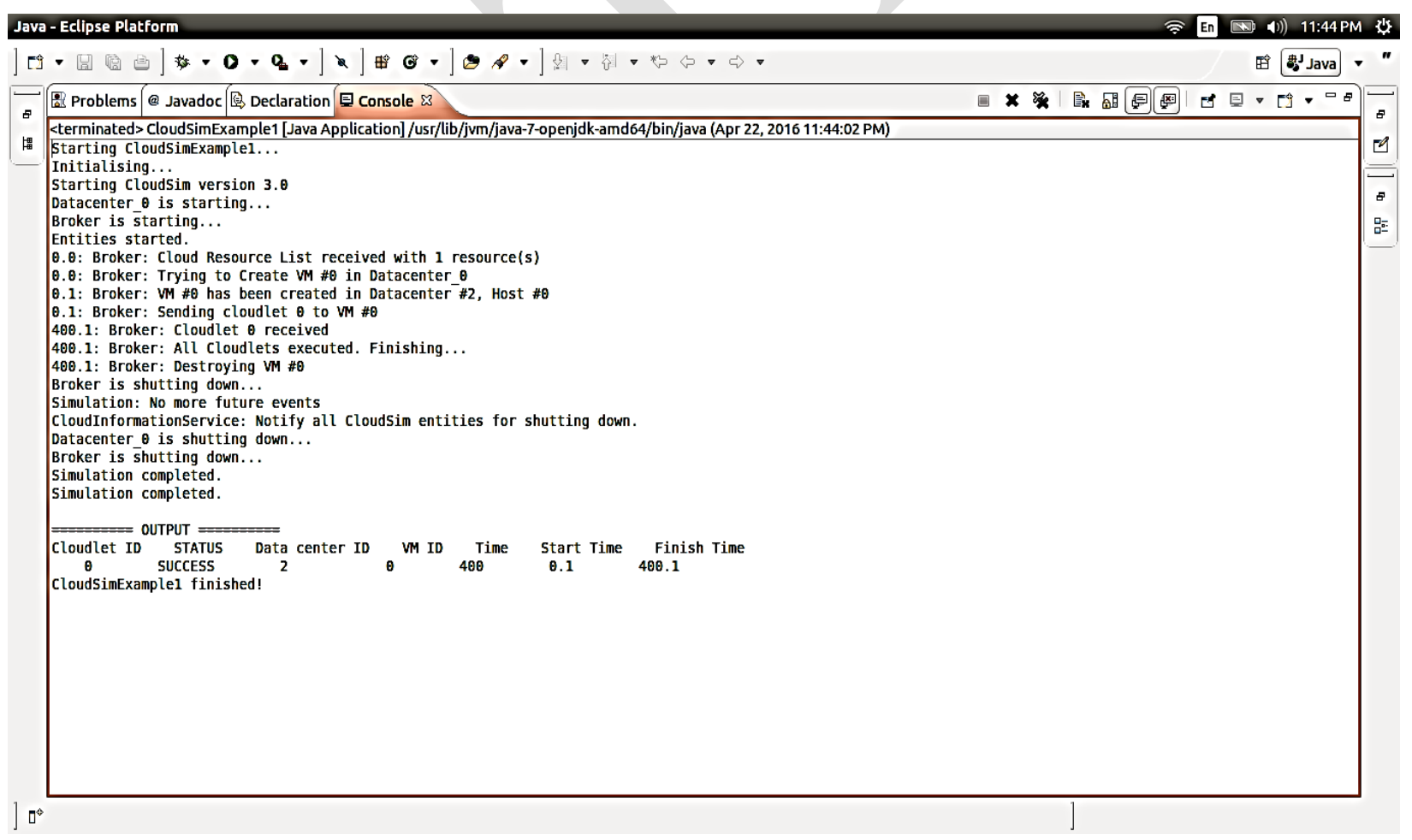

\section{Figure6 Experiment 1 output}

Above figure 6 shows a simple example 1 showing how to create a datacenter with one host and run one cloudlet on it.Cloudlet is nothing but task assigned to cloud. Id's are starting from 0 , output shows on datacenter Id 2 task i.e. cloudlet id 0 assigned and executed within start and finish time (in millisecond) as shown above. 


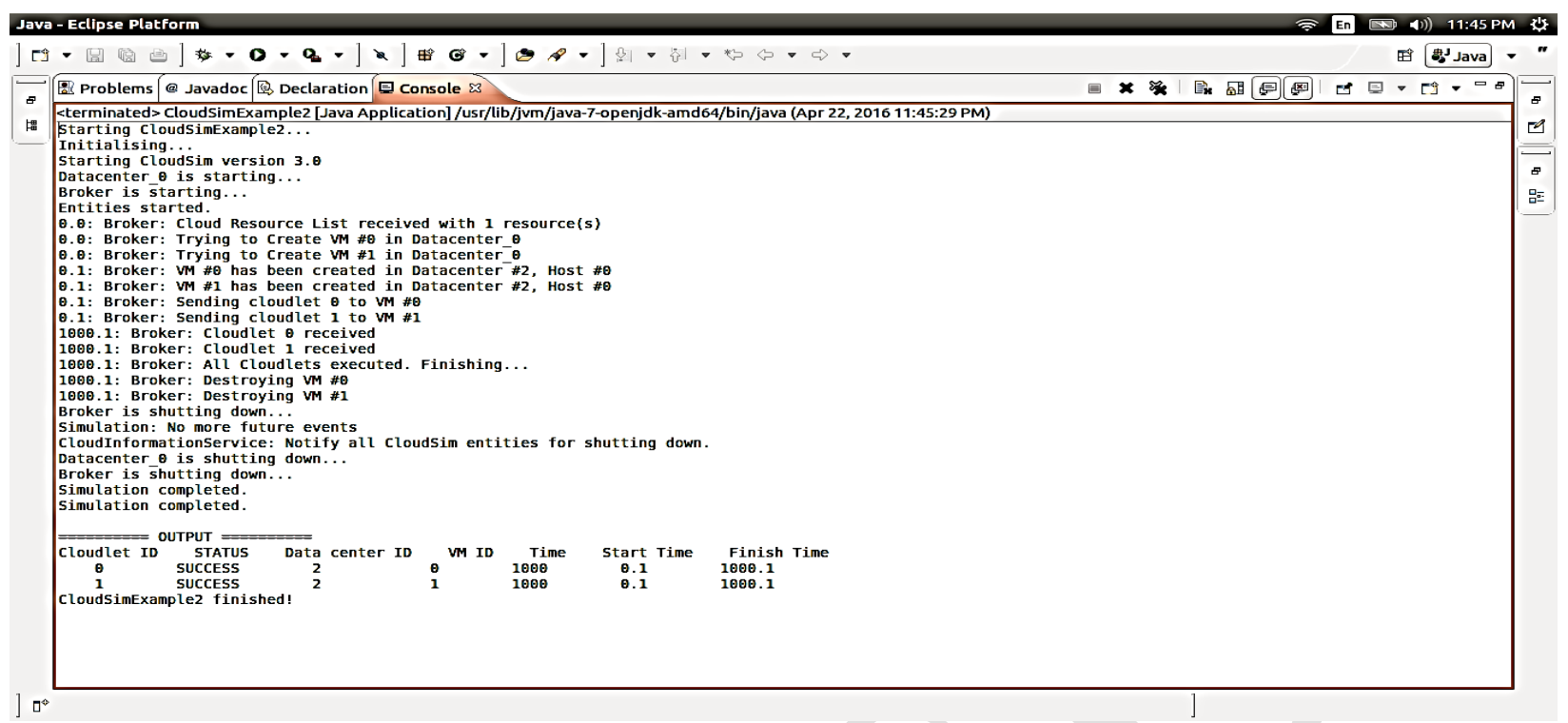

\section{Figure 7 Experiment 2 output}

Same like this experiment 2 carried out for showing how to create a datacenter with one host and run two cloudlets on it. The cloudlets run in VMs with the same MIPS requirements. The cloudlets will take the same time to complete the execution. Output of experiment 2 shown in above figure 7.

CloudSim start and after all execution of cloudlets shutdown automatically, it was observed in output that broker started checks for datacenter to execute cloudlet, assign it to respective datacenters respective virtual machines, executed the cloudlet and then after finishing all cloudlets destroyed virtual machines, shutting down the brokers, checks whether there are any future events for execution if no, notify that to all cloudsim entities that all work is done with shutting down all datacenter, broker and stopped the execution of simulation. Further power class can be modified to show the power consumption by respective datacenters.

Experiment 3 showing how to create a datacenter with two hosts and run two cloudlets on it. The cloudlets run in VMs with different MIPS requirements. The cloudlets will take different time to complete the execution depending on the requested VM performance, output shown below infigure 8 .

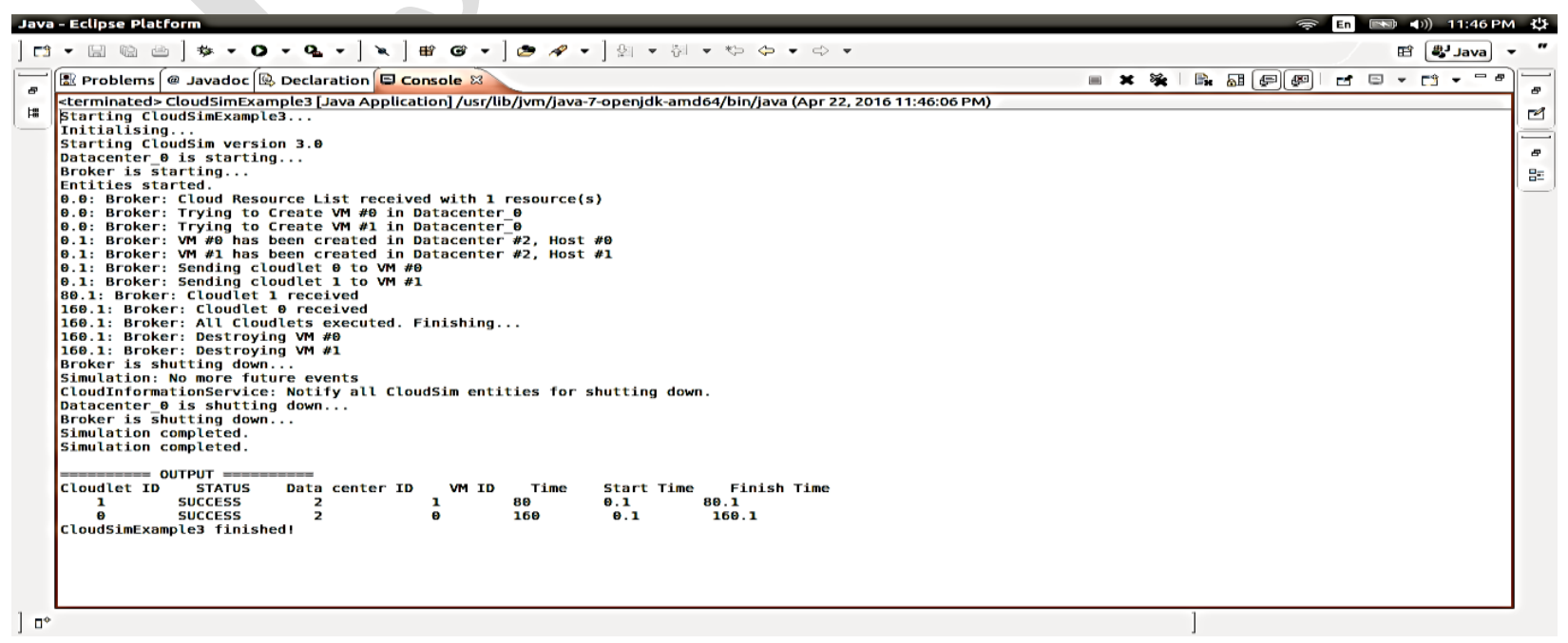

Figure 8 Experiment 3 output 
Experiment 4 was showing how to create two datacenters with one host each and run cloudlets of two users on them, output shown in figure 9.

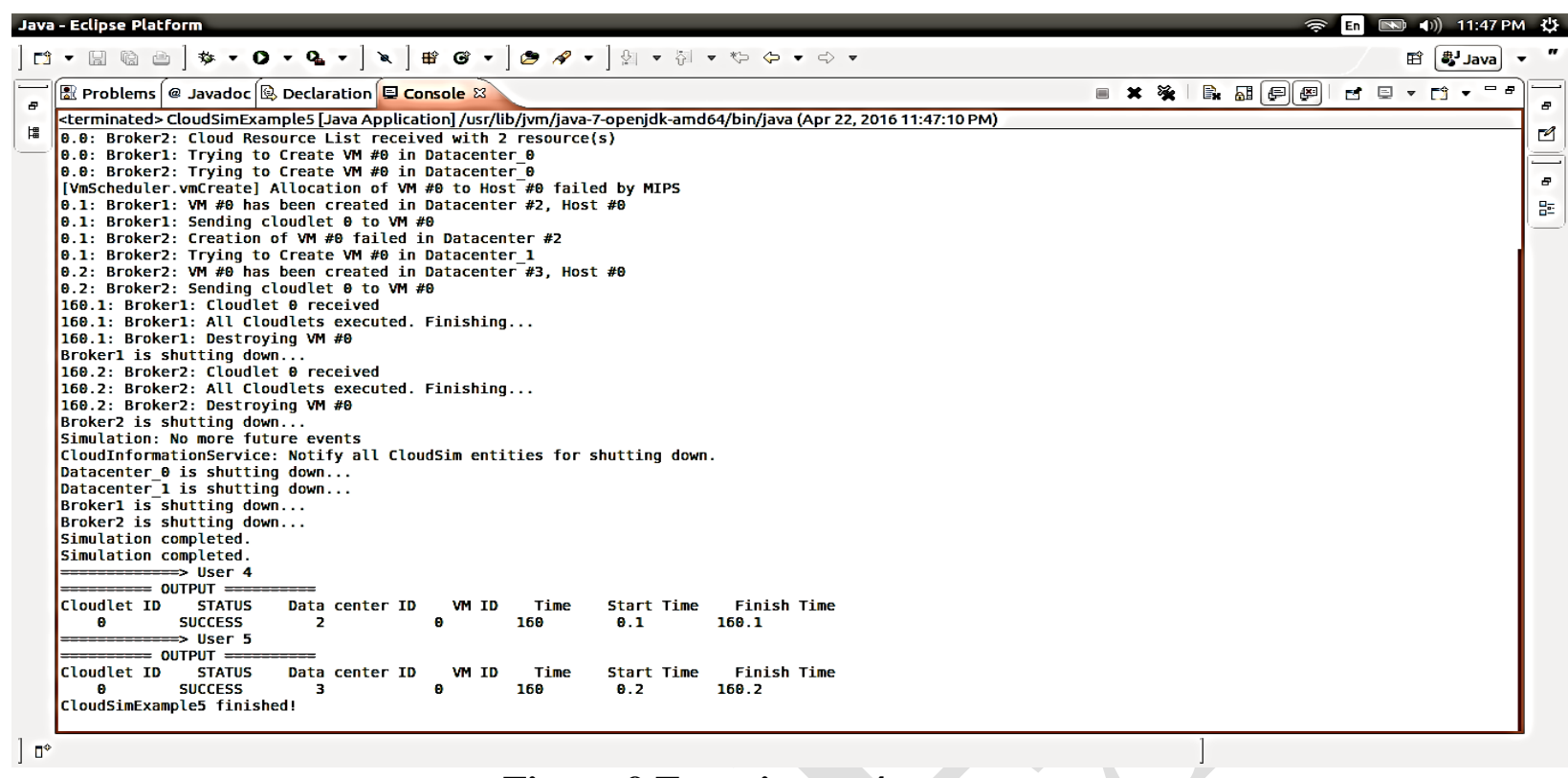

\section{Figure 9 Experiment 4 output}

\section{CONCLUSION}

Study of Aneka cloud along with its detail architecture and implementation for the intending mathematical model objective has observed foe energy efficient cloud andstudy of cloudsim which is used for modeling and simulation of cloud computing applications also experimented it for setting up datacenters and virtual machines with different multiple instruction per second (MIPS). Researchers will be very happy to use these simulators and get ahead with there researchers.

\section{REFERENCE}

I. Calheiros, Rodrigo N. et al. "Cloudsim: A Toolkit For Modeling And Simulation Of Cloud Computing Environments And Evaluation Of Resource Provisioning Algorithms". Softw: Pract. Exper. 41.1 (2010): 23-50. Web.

II. D. Chappell. Introducing the Azure services platform.White paper, Oct. 2008.

III. X. Chu et al. Aneka: Next-generation enterprise gridplatform for e-science and ebusiness applications.Proceedings of the 3rd IEEE InternationalConference on e-Science and Grid Computing, 2007.

IV.C. L. Dumitrescu and I. Foster.GangSim: a simulatorfor grid scheduling studies. Proceedings of the IEEEInternational Symposium on Cluster Computing andthe Grid, 2005.

V. I. Foster and C. Kesselman (editors). The Grid:Blueprint for a New Computing Infrastructure.Morgan Kaufmann, 1999.

VI. F. Howell and R. Mcnab.SimJava: A discrete eventsimulation library for java. Proceedings of the firstInternational Conference on Web-Based Modelingand Simulation, 1998.

VII. A. Legrand, L. Marchal, and H. Casanova. Schedulingdistributed applications: the SimGridsimulationframework. Proceedings of the 3rd IEEE/ACMInternational Symposium on Cluster Computing andthe Grid, 2003. 
VIII. J. E. Smith and R. Nair.Virtual Machines: Versatileplatforms for systems and processes. MorganKauffmann, 2005.

IX. R. Buyya and M. Murshed.GridSim: A Toolkit for theModeling and Simulation of Distributed ResourceManagement and Scheduling for Grid Computing.Concurrency and Computation: Practice andExperience, 14(13-15), Wiley Press, Nov.-Dec., 2002.

X. A. Weiss. Computing in the clouds.NetWorker,11(4):16-25, Dec. 2007.

XI. M. Armbrust, A. Fox, R. Griffith, A. Joseph, R. Katz, A.Konwinski, G. Lee, D. Patterson, A. Rabkin, I. Stoica,M. Zaharia. Above the Clouds: A Berkeley View of Cloudcomputing. Technical Report No. UCB/EECS-2009-28,University of California at Berkley, USA, Feb. 10, 2009.

XII. R. Ranjan and R. Buyya. Decentralized Overlay forFederation of Enterprise Clouds. Handbook of Researchon Scalable Computing Technologies, K. Li et. al. (ed),IGI Global, USA, 2009 (in press).

XIII. R. Buyya, C. S. Yeo, S. Venugopal, J. Broberg, and I.Brandic. Cloud Computing and Emerging IT Platforms:Vision, Hype, and Reality for Delivering Computing asthe 5th Utility. Future Generation Computer Systems,25(6): 599-616, Elsevier Science, Amsterdam, TheNetherlands, June 2009.

XIV. Kumar, Ranjan and G Sahoo. "Cloud Computing Simulation Using Cloudsim". IJETT 8.2 (2014): 82-86.

XV. Buyya, Rajkumar, Christian Vecchiola, and S. ThamaraiSelvi. Mastering Cloud Computing. Waltham, MA: Morgan Kaufmann, 2013. Print. 